\title{
Monitoring des metastasierten Nierenzellkarzinoms - Standards und Herausforderungen
}

\author{
Elke Jäger \\ Klinik für Onkologie und Hämatologie, Krankenhaus Nordwest, Frankfurt/M.
}

\author{
Schlüsselwörter \\ Nierenzellkarzinom $\cdot$ Monitoring $\cdot$ RECIST . \\ Tyrosinkinase-Hemmer · Sunitinib
}

\section{Zusammenfassung}

Durch die Einführung von Targeted Therapies hat sich für die Beurteilung des metastasierten Nierenzellkarzinoms (mRCC) eine völlig neue Situation ergeben: Die Patienten profitieren von den neuen Substanzen mit einem wesentlich längeren Überleben als noch vor einigen Jahren. Um diesen Nutzen der Therapie zu maximieren, stellen sich neue Anforderungen an die Verlaufsbeobachtung, insbesondere an die Bildgebung sowie die Interpretation der Befunde. Dies erfordert unter anderem genaue Kenntnisse des Wirkmechanismus der neuen Substanzen, da sie andere Veränderungen am Tumor hervorrufen als klassische Zytostatika. Standard für die Beurteilung sind die RECIST-Kriterien. In Anlehnung an die Empfehlungen der Interdisziplinären Arbeitsgruppe Nierenzellkarzinom der Deutschen Krebsgesellschaft (DKG) sollte bei zielgerichteten Therapien in jeder Therapielinie eine ausreichende Therapiedauer angestrebt werden, um einen optimalen Behandlungserfolg zu erzielen. Die Behandlung wird jeweils fortgesetzt bis zum Eintritt einer klinischen Progression. Um einen Progress auszuschließen, ist eine Kontrolle des Therapieerfolgs mittels adäquater bildgebender Verfahren rund 6-9 Wochen nach Beginn der Therapie sinnvoll. Danach sollten sich die Kontrolluntersuchungen im Intervall von 2-3 Monaten mit der gleichen Methodik wiederholen. Bei zyklisch applizierten Therapien wie Sunitinib sind bildgebende Untersuchungen jeweils zu gleichen Zeitpunkten innerhalb des Therapiezyklus angezeigt.

Neue Substanzen stellen neue Anforderungen an die Beurteilung des Therapieverlaufs

Die Etablierung zielgerichteter Substanzen in der Therapie des fortgeschrittenen/metastasierten Nierenzellkarzinoms (mRCC) hat verglichen mit historischen Standards - zu einer deutlichen Verlängerung des krankheitsfreien und des Gesamtüberlebens (Overall Survi-

\section{Key Words}

Renal cell carcinoma - Monitoring · RECIST .

Tyrosine kinase inhibitors $\cdot$ Sunitinib

\section{Summary}

Monitoring of Metastatic Renal Cell Carcinoma - Standards and Challenges

The introduction of targeted therapies has led to a novel situation regarding monitoring of metastatic renal cell carcinoma (mRCC): patients treated with these new drugs have a significantly longer life expectancy than just a few years ago. In order to maximize the treatment benefit, new demands have come up on the assessment of tumor progression: timimg and interpretation of imaging studies are crucial for optimal therapeutic management. Detailed knowledge of the new compounds' mode of action is important as it is different from that of classic cytotoxic drugs. The RECIST criteria constitute the standard for evaluation. Following recommendations made by the German Cancer Society's (DKG) interdisciplinary task force, targeted therapies should strive for a sufficient treatment duration in each line of therapy in order to achieve the best therapeutic outcome. Treatment is continued until clinical progression occurs. Assessing therapeutic efficacy with adequate imaging techniques 6-9 weeks after the beginning of therapy is recommended. Subsequent follow-up examinations should be repeated using identical imaging modalities every 2-3 months. For cyclically applied drugs such as Sunitinib, examinations should be carried out at identical time points within the treatment cycle.

\begin{tabular}{ll}
\hline KARGER & @ 2010 S. Karger GmbH, Freiburg \\
Fax +497614520714 & Accessible online at: \\
Information@Karger.de & www.karger.com/onk \\
www.karger.com &
\end{tabular}


konventionelle Zytostatika. Darüber hinaus werden RCC-Patienten aufgrund der geringen Inzidenz dieses Tumors insgesamt selten beim Radiologen vorstellig. Diese Aspekte können hohe qualitative Anforderungen an das Monitoring, sowohl hinsichtlich der Bildgebung als auch der Interpretation der Befunde stellen.

\section{Standard RECIST}

Grundsätzlich erfolgt die Verlaufsbeobachtung beim mRCC in enger Zusammenarbeit von Urologen, Onkologen und Radiologen. Im metastasierten Stadium der Erkrankung hat das radiologische Therapiemonitoring einen besonders hohen Stellenwert. Standard für die Auswertung und Beurteilung sind die RECIST(response evaluation criteria in solid tumors)-Kriterien [3]. Die Definitionen für ein Ansprechen nach RECIST sind in Abbildung 1 dargestellt. Es gibt verschiedene Ansätze, diese Kriterien zu präzisieren [4], auch mittels neuer softwarebasierter volumetrischer Methoden [5].

Besonderheiten des Monitorings unter der Therapie mit Sunitinib

In der Zulassungsstudie hatten 47\% der Patienten unter Sunitinib eine Tumorregression nach RECIST (CR + PR), 40\% erreichten eine Krankheitsstabilisierung (SD) [1]. Grundsätzlich wird empfohlen, die Therapie möglichst lange fortzuführen, da sich gerade bei Sunitinib gezeigt hat, dass die Rate der radiologischen Remissionen $(\mathrm{CR}+\mathrm{PR})$ im Therapieverlauf zunimmt $[1,2,6]$. In der

\section{Ansprechen nach RECIST}

\begin{tabular}{|l|l|}
\hline Komplette Remission (CR) & Verschwinden aller Läsionen \\
\hline Partielle Remission (PR) & $\begin{array}{l}\geq 30 \% \text { Reduktion des längsten } \\
\text { Durchmessers der Läsion }\end{array}$ \\
\hline Stabile Erkrankung (SD) & $\begin{array}{l}\text { Patient erfüllt weder die Kriterien } \\
\text { für die PR noch für eine Progression }\end{array}$ \\
\hline Progression (PD) & $\begin{array}{l}\geq 20 \% \text { Zunahme der Tumorgröße } \\
\text { in der Summe der längsten } \\
\text { Diameter verglichen mit dem } \\
\text { besten Ansprechen oder Auftreten } \\
\text { einer neuen Läsion }\end{array}$ \\
\hline $\begin{array}{l}\text { Eisenhauer EA, Therasse P, Bogaerts J et al. New response evaluation criteria } \\
\text { in solid tumours: Revised RECIST guideline (v1.1). Eur 3 Cancer 2009; 45:228-47. }\end{array}$ \\
\hline
\end{tabular}

Abb. 1. Die RECIST-Kriterien definieren das Ansprechen bei soliden Tumoren. Sie gelten als Standard bei der Beurteilung radiologischer Befunde.

klinischen Praxis stellt sich häufig die Frage, ob man nach Erreichen einer Remission die Therapie fortsetzen soll, insbesondere dann, wenn es sich um eine komplette Remission handelt [7]. Für eine Fortsetzung spricht zum einen die Überlegung, dass grundsätzlich nur unter fortgesetzter Angiogenesehemmung ein andauernder Therapieerfolg sichergestellt werden kann, zum anderen die Beobachtung, dass die Erkrankung nach Absetzen der Therapie häufig wieder progredient wird [7]. Gegenwärtig kann allen-

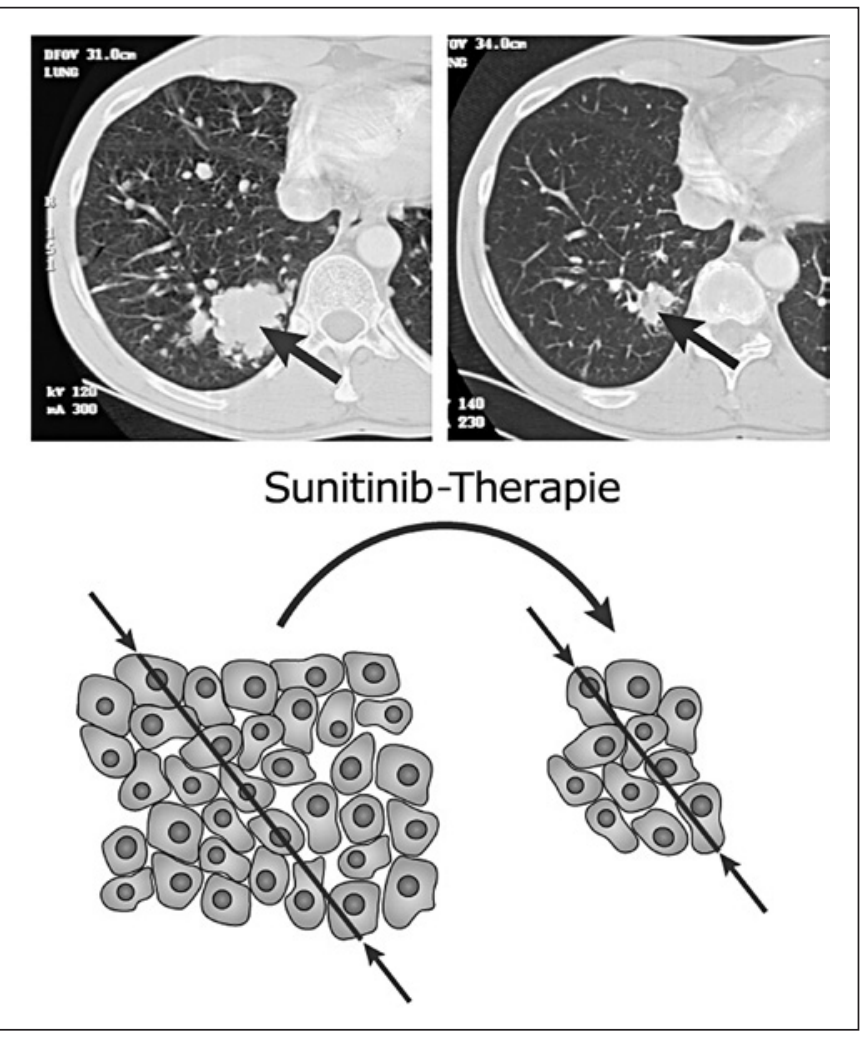

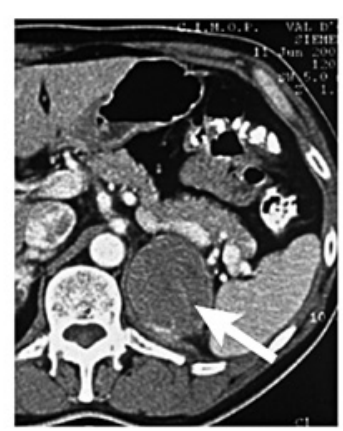

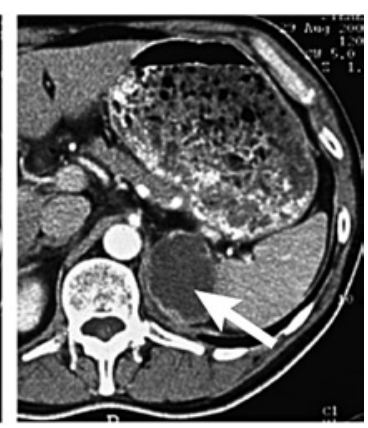

Sunitinib-Therapie

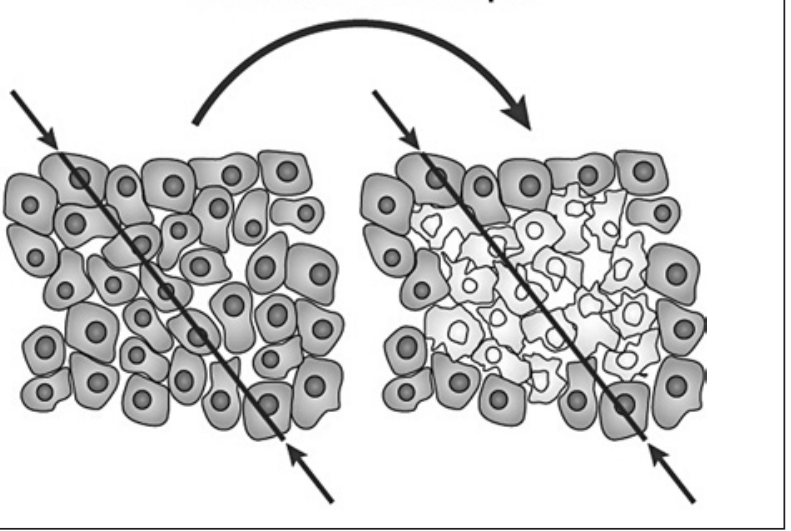

Abb. 2. Effekte von Sunitinib auf Angiogenese und Tumorzellen: Therapieeffekte durch Reduktion der Tumorgröße (links) bzw. Tumornekrose (rechts) [8]. 
falls nach Erreichen einer kompletten Remission ein Absetzen der Therapie im Einzelfall erwogen werden. Die Abwägung sollte unter enger Einbeziehung des Patienten bzw. dessen Angehörigen und unter regelmäßiger bildgebender Rezidiv-Kontrolle erfolgen. Umgekehrt sollte jedoch auch eine vermeintliche Wirkungslosigkeit nicht zur verfrühten Beendigung der Therapie führen.

\section{Effekte der anti-angiogenetischen Therapie}

Aufgrund des anti-angiogenetischen Wirkmechanismus kann es zu Beginn der Therapie sogar zu einer Größenzunahme des Tumors in der Bildgebung kommen, da Nekrosen im Inneren zum Aufquellen des Tumors führen können. Weitere mögliche Effekte auf den Tumor sind eine verringerte Tumordurchblutung und eine reduzierte Tumordichte. Eine Stabilisierung ohne Größenveränderung stellt einen Therapieerfolg dar, auch eine signifikante Reduktion der Tumorgröße ist möglich (Abb. 2).

Da die Therapie mit Sunitinib nach dem 4/2-Schema (4 Wochen Therapie, danach 2 Wochen Therapiepause) erfolgt, muss zusätzlich berücksichtigt werden, dass sich diese zyklische Hemmung des Tumors auch in einem kurzfristigen radiologischen Rebound in der Therapiepause niederschlagen kann.

\section{Empfehlungen der Arbeitsgruppe Nierenzellkarzinom der Deutschen Krebsgesellschaft}

Auf Basis der aktuellen Daten und unter Berücksichtigung der spezifischen Therapiesituation unter Tyrosinkinase-Inhibitoren (TKI) hat die Interdisziplinäre Arbeitsgruppe Nierenzellkarzinom der Deut- schen Krebsgesellschaft (DKG) folgende Hinweise zur Verlaufskontrolle beim RCC verabschiedet [6]:

- Eine Kontrolle des Therapieerfolgs mittels adäquater bildgebender Verfahren ist rund 6-9 Wochen nach Beginn der Therapie sinnvoll (je nach Metastasenlokalisation CT oder MRT), um einen Progress auszuschließen. Danach sollten sich die Kontrolluntersuchungen im Intervall von 2-3 Monaten nach Möglichkeit mit der gleichen Methodik wiederholen. Bei zyklisch applizierten Therapien, wie Sunitinib, sollten bildgebende Untersuchungen jeweils zu gleichen Zeitpunkten innerhalb des Zyklus erfolgen.

- Die Therapie bzw. Therapiesequenz wird jeweils bis zum Eintritt einer Progression oder therapielimitierenden Nebenwirkungen durchgeführt. Bei gemischtem Ansprechen kann die Therapie fortgesetzt werden, solange der Patient davon einen klinischen Nutzen hat.

- Bei zielgerichteten Therapien sollte in jeder Therapielinie eine ausreichende Therapiedauer angestrebt werden, um einen optimalen Behandlungserfolg zu erzielen. Jede Behandlung wird jeweils fortgeführt bis zum Eintritt einer klinischen Progression (radiologisch bestätigte Progression oder Verschlechterung des klinischen Bildes bzw. bis zum Auftreten von intolerablen Nebenwirkungen). Zur Beurteilung der radiologischen Befunde sollten die aktualisierten RECIST-Kriterien herangezogen werden. Wie lange eine Therapie nach Erreichen einer Komplettremission unter der Therapie mit TKI zur Vermeidung eines Rezidivs fortgeführt werden sollte, ist nach derzeitiger Datenlage unklar.

\section{Disclosure Statement}

The author is a member of the advisory boards and lecturer for Pfizer, Roche, Bayer, GlaxoSmithKline, Lilly and participates in clinical trials funded by Pfizer, Novartis, Wyeth, Sanofi, and GlaxoSmithKline.

\section{Literatur}

1 Motzer RJ, Hutson TE, Tomczak P, et al: Sunitinib versus interferon alfa in metastatic renal-cell carcinoma. N Engl J Med 2007;56:115-124.

2 Figlin RA, Hutson TE, Tomczak P, et al: Overall survival with sunitinib versus interferon (IFN) alfa as first-line treatment of metastatic renal cell carcinoma (mRCC). J Clin Oncol 2008;26(suppl):abstr 5024.

3 Eisenhauer EA, Therasse P, Bogaerts J, et al: New response evaluation criteria in solid tumours: Revised RECIST guideline (version 1.1). Eur J Cancer 2009;45:228-247.
4 Choi H, Charnsangavej C, Faria SC, et al: Correlation of computed tomography and positron emission tomography in patients with metastatic gastrointestinal stromal tumor treated at a single institution with imatinib mesylate: proposal of new computed tomography response criteria. J Clin Oncol 2007;25:1753-1759.

5 Graser A, Becker CR, Reiser M, et al: Volumetrie von Metastasen beim Nierenzellkarzinom. Radiologe 2008;48:850-856.

6 Interdisziplinäres Expertengespräch Nierenzellkarzinom der Deutschen Krebsgesellschaft 2009. www. krebsgesellschaft.de/expertengespraech_rcc_2009.
7 Johannsen M, Flörcken A, Bex A, et al: Can tyrosine kinase inhibitors be discontinued in patients with metastatic renal cell carcinoma and a complete response to treatment? A multicentre, retrospective analysis. Eur Urol 2009;55:1430-1439.

8 Faivre S, Demetri G, Sargent W, et al: Molecular basis for sunitinib efficacy and future clinical development. Nat Rev Drug Discov 2007;6:734-745. 